\title{
Ein Fall von Sarcoma uteri-deciduocellulare.
}

\author{
Von \\ Dr. Tannen,
}

Frauenarzt in Hannover.

(Mit 2 Abbildurgen auf $\mathrm{Tafel}$ IV.)

Durch die Arbeiten Gottschalk's') und Sänger's ${ }^{2}$ ) wurde diese bis dahin fast unbekannte Krankheit der Vergessenheit entrissen und die bis dahin veröffentlichten Fälle wurden kritisch durch Sänger geprüft. Wenn auch mehrere der beschriebenen Fälle, wie Sänger nachweist, sieher nicht zu diesem Namen berechtigt sind, so geht doch aus allen Mittheilungen hervor, dass die Erkrankung eine seltene und eine höchst gefährliche ist. Alle Kranke starben, selbst die Gottschalk's, bei der es zum ersten Male gelang, durch mikroskopische Untersuchung curettirter Massen die wissenschaftliche Diagnose an der Lebenden zu stellen und darauf hin mit Erfolg den Uterus zu entfernen, erlag 7 Monate nach der Operation ihrem Leiden. Löhlein ${ }^{3}$ ) war der zweite, der die Diagnose an der Lebenden durch die mikroskopische Untersuchung feststellen und die Kranke radikal operiren konnte. Aber auch diese Patientin ist später gestorben und wahrscheinlich an einem Recidiv. $\mathrm{Ob}$ in dem Fall von Löhlein wirklich dieselbe Geschwulstform vorlag, die bei Gottschalk und den Anderen als Deciduoma malignum bezeichnet wird, will ich nicht erörtern. Zur Zeit ist der klinische Begriff "Sarcoma deciduo-cellulare" wohl fest-

1) Gottschalk, Ueber das Sarcoma chorio-deciduo-cellulare. Berl. klin. Wochensehr., 1893, No. 4 u. 5.

2) Sänger, Ueber Sarcoma uteri-cellulare und andere deciduale Geschwülste. Dieses Archiv, Bd. 44, H. 1.

3) Löhlein, Sarcoma deciduo-cellulare nach vorausgegangenem Myxoma chorii. Centralbl. f. Gyn., 1893, No. 14. 
stehend und klar, die phatologisch-anatomische Definition dieser eigenartigen Geschwulstform dagegen jetzt noch verworren und unklar. Erst ein grösseres mikroskopisch genau durchgearbeitetes Material wird darin Klarheit bringen und wahrscheinlich den klinischen SammeInamen "Sarcoma deciduo-cellulare" wieder in einzelne Unterabtheilungen zergliedern, die sich nicht klinisch aber: wohl histologisch und vielleicht auch prognostisch trennen lassen. Der dritte Fall, der in vivo dureh das Mikroskop diagnosticirt und darauf radikal operirt wurde, ist der von Nové-Josserand und Lacroix ${ }^{1}$. Aehnlich wie bei der Kranken Löhlein's war auch hier früher eine Blasenmole vorhanden gewesen. Die letzte mir bekannte Untersuchung dieser Patientin 3 Monate nach der Totalexstirpation ergab völlige Recidivfreiheit. Der Fall Paviots²), eine Combination von Deciduom und gutartigem, glandulärem, cystischem Adenom des Uterus bei einer Kranken, die übrigens nicht geboren hatte und bereits 48 Jahre alt war, kam nicht zur Operation. Die Kranke aus der Zweifel'sehen ${ }^{3}$ ) Klinik ist wohl die 4., die operirt wurde. Auch sie starb 6 Monate nach der Totalexstirpation an Metastasen. Der Fall aus der Münchener") Frauenklinik erlag unoperirt einer heftigen Blutung. Weitere Veröffentlichungen ${ }^{5}$ ) hierüber liegen meines Wissens nicht vor. Es ist wohl selbstverständlich, dass die Krankheit nicht so selten ist wie die Litteratur zeigt, schon jetzt mehren sich die Beiträge, aber Gott sei Dank, scheint sie doch nicht häufig vorzukommen.

Wenn ich nun im Folgenden die Krankengeschichte eines von mir operirten Falles etwas ausführlicher mittheile, so geschieht das aus einem späterhin zu erwähnenden Grunde.

Frau F. aus Hannover, 23 Jahr alt, will immer gesund gewesen sein und regelmässig menstruirt haben. Sie ist seit 5 Jahren verheirathet. Die erste Entbindung erfolgte vor 4 Jahren spontan, die zweite

1) Nové-Josserand und Lacroix, Sur le déciduome. Annales de Gynécologie et d'obstétrique. Februar, März, April 1894.

2) Paviot, Un cas de décidnome malin avec noyaux métastatiques multiples. Ibidem. April 1894.

3) Menge, Centralbl. f. Gyn., 1894, No. 11, S. 264.

4) Klien, Ein Fall von Deciduo-Sarcoma uteri giganto-cellulare. Dieses Archiv, Bd. 47, H. 2.

5) Anmerk. nach der Correctur. Die nach Absendung dieser Mittheilung in der Literatur erschienenen Arbeiten sind hier nicht mehr berücksichtigt worden. 
Schwangerschaft endete im VI. Monat mit einem Abort. Die 3. Schwangerschaft, im Sommer 1893, auffallend durch sehr häufiges, ihr sonst unbekanntes Erbrechen, Drängen nach unten, endete nach 3 monatlichem Bestehen im Juli 1893 mit der Ausstossung einer Blasenmole in Gegenwart ihres Hausarztes, Dr, Giese. Die Blutung dauerte 3 Wochen und stand auf Secale. Von jetzt an menstruirte sie regelmässig in 3 wöchentlichen Zwischenpausen und fühlte sich ganz gesund, - Ende Januar 1894 trat die Periode zuletzt auf, bis Anfang März sich Blutungen einstellten. Mit dem Beginn derselben stellte sich auch eine auffallende Mattigkeit ein, die sie, ebenso wie die fortwährenden blutigen Ausflüsse beunruhigten. Ám 28. April wurde ich von dem Collegen zugezogen. Die Untersuchung ergab eine mässige Anaemie, einen dem Anfang des 3. Schwangerschaftsmonates an Grösse entsprechenden Uterus, der retrovertirt lag und den Finger nur bis zum Orif. int. durchliess. In Chloroformnarcose wurde der Uterus mit Celluloid-Dilatatorien erweitert, ausgespült, curettirt, wieder ausgespült, mit Ac. carb. pur. liq. ausgeätzt und mit Jodoformgaze austamponirt. Die entfernten für Abortreste gehaltenen Massen wurden nicht weiter beachtet. Zuerst ging es der Kranken gut, aber dann stellten sich übelriechender, blutiger Ausfluss, Fieber und anhaltendes, sehr quälendes Erbrechen ein, wodurch die Kranke sehr herunterkam. Bei einer erneuten Consultation am 22. Mai musste ich zu meinem grossen. Erstannen und Aerger feststellen, dass der Uterus zu gross und das Orif. ext. für einen Finger durchgängig war, mithin wahrscheinlich noch Abortreste von mir zurückgelassen waren. Da aber möglicherweise die Retroflexio und eine dadurch bedingte Retention der Sekrete Ursache des jauchigen Ausflusses und einer im rechten Parametrium entstandenen Infiltration sein konnte, stand ich von dem Curettement ab, reponirte den Uterus durch ein Pessar und spülte ihn aus. Am 24. Mai erfolgte eine nochmalige Uterusausspülung, nachdem am Tage vorher der Hausarzt dies besorgt hatte. Am 9. Juni wieder zugezogen, erfuhr ich, dass Fleischstïcke abgegangen seien, die Kranke hoch fiebere, alles erbreche und rapide abmagere. Ich fand nun ein rechtsseitiges parametritisches Exsudat, dem ich das Erbrechen, Fieber ete. zuschob. Als am 16. Juni der Zustand sich immer mehr verschlechterte, schlug ich, wenn auch ungern, ein erneutes Curettement vor, das auch Abends mit der Wanscher'schen Maske in Aethernarcose in derselben Weise wie vorher ausgeführt wurde. Den Aether wäblte ich in Hinsicht auf den frequenten kleinen Puls und die grosse Anaemie und Schwäche. Als bei dem Curettement wieder 2-3 Esslöffel weicher hochrother Massen entfernt wurden und der eingeführte Finger im Fundus eine weiche, schwammige Masse fühlte, dachte ich sofort an das maligne Deciduom, da ich mir so viel Ungeschicklichkeit, beim ersten Curettement so viel zurückgelassen zu haben, nicht zutraute. Den Verdacht äusserte ich auch beim Weggange dem Hausarzte. Das Curettirte wurde in Spiritus gelegt und die Hälfte meinem früheren Mitassistenten, jetzigen Privatdocenten in Breslau, Herrn Dr. Pfannenstiel, dessen Erfahrung und Uebung in mikroskopischen Untersuchungen ich zur Genüge kannte, zugesandt. Die Untersuchung der in Celloidinlösung eingeschlossenen und auf dem Mikrotom geschnittenen Gewebsstücke ergab nach Färbung: grosse Deciduazellen von der bekannten Form ohne besondere Anordnung als z. B. alveolärer Bau, blutige Extravasate und Fibrin verschiedenen Alters, daneben auch vereinzelte Zellen, die im Uebergangsstadium von Deciduazellen zu Rund- und Spindelzellen zu sein schienen, 
endlich Streifen von Deciduazellen zwischen musculösen Elementen, die durch diese grossen Zellen förmlich auseinandergedrängt waren. Letzteres war für mich maassgebend und bestimmend, die Diagnose auf Sarcoma deciduo-cellulare zu stellen.

Da das Resultat des Breslaner Collegen noch mebrere Tage auf sich warten liess, theilte ich nur dem Hausarzt mein Ergebniss mit, weil ich persönlich nie einen derartigen Tumor gesehen hatte und nicht allein die Verantwortung des mikroskopischen Untersuchungsresultates tragen wollte. Inzwischen musste noch eine Frage nothwendig erledigt werden. War die Infiltration des rechten Parametrium eine gewöhnliche Parametritis, also eine gutartige oder eine bösartige, d. h. eine Infection des Parametrium und des Ligamentes mit Tumormassen? War Letzteres sicher, hatte eine Operation natürlich keinen Sinn. Es lag ja nahe, daran zu denken, zumal heftige Schmerzen in der Leber sofort den Gedanken an Metastasen wachriefen.

Am 25. Juni punctirte ich in Aethernareose in der Wohnung der Kranken an verschiedenen Stellen, ohne Eiter zu finden. Dadurch wurde die Möglichkeit, dass die Infiltration eine gutartige wäre, noch unwahrscheinlicher. Allein eine, wenn auch geringe Möglichkeit der Gutartigkeit dieser Infiltration war doeh noch rorhanden und sie bewog mich, der Familie die Totalexstirpation doch vorzuschlagen, allerdings mit dem Bemerken, dass die Chancen der Operation selbst in Rüicksicht auf die Abmagerung, den schlechten Puls, das fortwährende Erbrechen schlechte seien und dass die Gefahr eines Recidives eher wahrscheinlich sei als unwahrscheinlich. Der Gedanke jedoch, dass die junge Frau ohne Operation unrettbar verloren sei, siegte und bestimmte die Kranke selbst und ihre Angehörigen zur Operation. Sehr erfreulich für mich und meine Verantwortung war das etwas spät eingetroffene Resultat des Collegen Pfannenstiel, der dieselbe Diagnose stellte und auch zur Operation rieth.

Am 30. Juni entfernte ich im Vincenzstift in Aethernarcose ohne Mühe den Uterus mittelst 6 Klammern, die ich mir zufällig einige Wochen vorher aus Brüssel hatte kommen lassen, und die ich hier zum ersten Male anstatt des von mir bisher angewandten Ligaturverfahrens gebrauchte. Ein Blutverlust fand nicht statt, da auch der Scheidenschnitt mit Paquelin ausgeführt wurde. Die Blase wurde vollständig vom Cterus und den Ligamenten - Mackenrodt's Beschreibung der Blase zu $2 / 3$ vor den Ligamenten und zu $1 / 3$ vor dem Uterus, kann ich nur zustimmen - abgelöst, der Douglas'sche Raum eröffnet, der Fundus mit scharfen Haken und Muzeux durch den Schlitz gezogen und jetzt wurden unter Controlle der Augen einfach mit je 3 Klammern die Ligamente und Parametrien abgequetscht und der Uterus entfernt. Ein Fächertampon verschloss die Bauchhöhle und der Rest derselben wurde um die Klammern gewickelt, um die Scheide vor dem Druck der Instrumente zu schützen. Ich möchte bezweifeln, ob die Kranke den beim Ligaturverfahren nicht zu vermeidenden Blutverlust überstanden hätte. Seitdem habe ich noch einige Uteri mittelst Klammern exstirpirt und habe vorläufig das Nähen bei der Exstirpation aufgegeben. Am Tage nach der Operation hörten Fieber, Erbrechen - und unerklärlicher Weise auch die Schmerzen in der Leber definitiv auf. Nach 48 Stunden erfolgte die Entfernung der Pincetten ohne Mühe im Bett. Die Kranke erholte sich so rasch und bekam einen solchen Heisshunger, dass man wirklich staunen musste ïber die gewaltige Aenderung, welche die Ent- 
fernung des Uterus bewirkte. Die Secretion war sehr reichlich und konnte nach Entfernung des Tampons am 8. Tage dureh Ausspülungen bald beseitigt werden.

Der Tumor im Fundus uteri hatte eine Farbe wie das Innere der Blutapfelsinen - Sänger nennt sie „rubinroth, wie ein Granatapfel", Nové-Josserand bezeichnet sie als nde coleration rouge sombre" - reichte an einer Stelle (a) bis dicht an die Serosa des Peritoneums (Taf. IV, Fig. 1) ${ }^{1}$, war aber noch überall von Muskelgewebe, das durch seine blasse Farbe sich scharf abhob von dem tiefrothen Tumorgewebe, umgeben. Dadurch schon wurde die Gutartigkeit der genannten Infiltration des Parametrium sebr wahrscheinlich. Beweisend wurde aber hierfür das stetige Zurückgehen des Infiltrates, das $21 / 2$ Monate nach der Operation nur noch strangförmig sich anfühlte. Um diese Zeit hatte sie 20 Pfund zugenommen. Jetzt 7 Monate nach der Operation ${ }^{2}$ ) ist sie noch ohne Recidiv und vollständig gesund. $\mathrm{Ob}$ sie es bleiben wird, kann ja Niemand sagen, da darüber keine Erfahrungen vorliegen. Sie ist also die 5., welche radikal operirt ist und zur Zeit die zweite, die meines Wissens noch lebt.

Taf. IV, Fig. 2, zeigt das mikroskopische Bild an der Grenze zwischen Tumor und Uterusmuskulatur. Das Eindringen der Deciduazellen zwischen die Muskelfasern, ihre verschiedene äussere Form, von der Spindelform bis zur vielkernigen Riesenzelle (g), sind in diesem Bild namentlich bei schwacher Vergrösserung deutlich zu sehen. Ein ähnliches Bild zeigte die mikroskopische Untersuchung der curettirten Massen vor der Operation, nur waren hier noch grosse Blutextravasate verschiedenen Alters vorhanden, ausserdem war die Färbung wegen der Necrose und Jauchung der betreffenden Gewebe nicht so scharf wie in dem Schnitt (Fig. 2) durch eine Partie des Tumors aus dem exstirpirten Uterus.

Die klinische Diagnose stützt sich auf den Befund und die Anamnese einer Schwangerschaft, auf die unregelmässigen Blutungen, die zuerst blutig serös, später übelriechend werden und dann mit Fieber verbunden sind, auf das Erbrechen, die Anaemie und und die Abmagerung, endlich auf den Misserfolg einer manuellen

1) Die beiden Zeichnungen verdanke ich der Liebenswürdigkeit meines hiesigen Specialcollegen, Dr. Odenthal, wofür ich ihm auch an dieser Stelle meinen Dank ausspreche.

2) Auch jetzt beim Durchlesen der Correctur - über 9 Monate nach der Operation - ist sie noch recidivfrei. 
oder instrumentellen Ausräumung. Dass ein Deciduom sofort ohne diesen Misserfolg diagnosticirt wird, bildet wohl immer die Ausnahme. Die digitale Austastung des Cavum uteri wird wohl das Vorhandensein von weichen Massen feststellen können, aber die Natur dieser durch den Finger zu bestimmen, halte ich für unmöglich, da die Tumormassen sich geradeso anfühlen wie Abortreste. Das jugendliche Alter, eine frühere Molenschwangerschaft sind zu verwerthen, aber nicht nothwendig. Ich habe den Eindruck, als ob in Zukunft diese Tumoren nach früherer Molenschwangerschft sich von denen, wo eine solche nicht vorausgegangen war, histologisch trennen lassen. Die Curette kann leicht den Uterus perforiren, worauf zu achten ist. Den Ausschlag giebt die mikroskopische Untersuchung der entfernten Massen. Diese müssen unter allen Umständen gehörig gehärtet, eingebettet, geschnitten und gefärbt werden. Zu kleine Schnitte können eventuell nur Tumorgewebe enthalten und zu falschen Schlüssen führen. Nicht die Deciduazellen an und für sich, nicht ihr eventueller Uebergang in Spindel- oder Rundzellen, nicht die Blutextravasate und die Fibrinbildungen sind für den Praktiker maassgebend, sondern allein das Hineinwuchern dieser Zellen zwischen die glatten Muskelfasern beweist ihre Malignität und ist erforderlich für die Indication zur Uterusexstirpation. Und so ist es gewiss nicht immer leicht, die Diagnose dieser eigenartigen Geschwulst, die vortrefflich den Uebergang von normalem Gewebe in ein krankhaftes, bösartiges illustrirt, zu stellen. Diesem Uebelstande kommt die klinische Diagnose mit den erwähnten Momenten zu Hülfe. Und gar manche Krankengeschichte wird ganz oder sehr ähnlich der bier mitgetheilten Jauten, gar mancher Uterusexstirpation wegen Sarcoma uteri deciduo-cellulare wird eine ähnliche Vorgeschichte wie diese oder wie die ron Gottschalk vorangehen. Und deshalb hielt ich eine etwas ausführlichere Mittheilung derselben für berechtigt. 

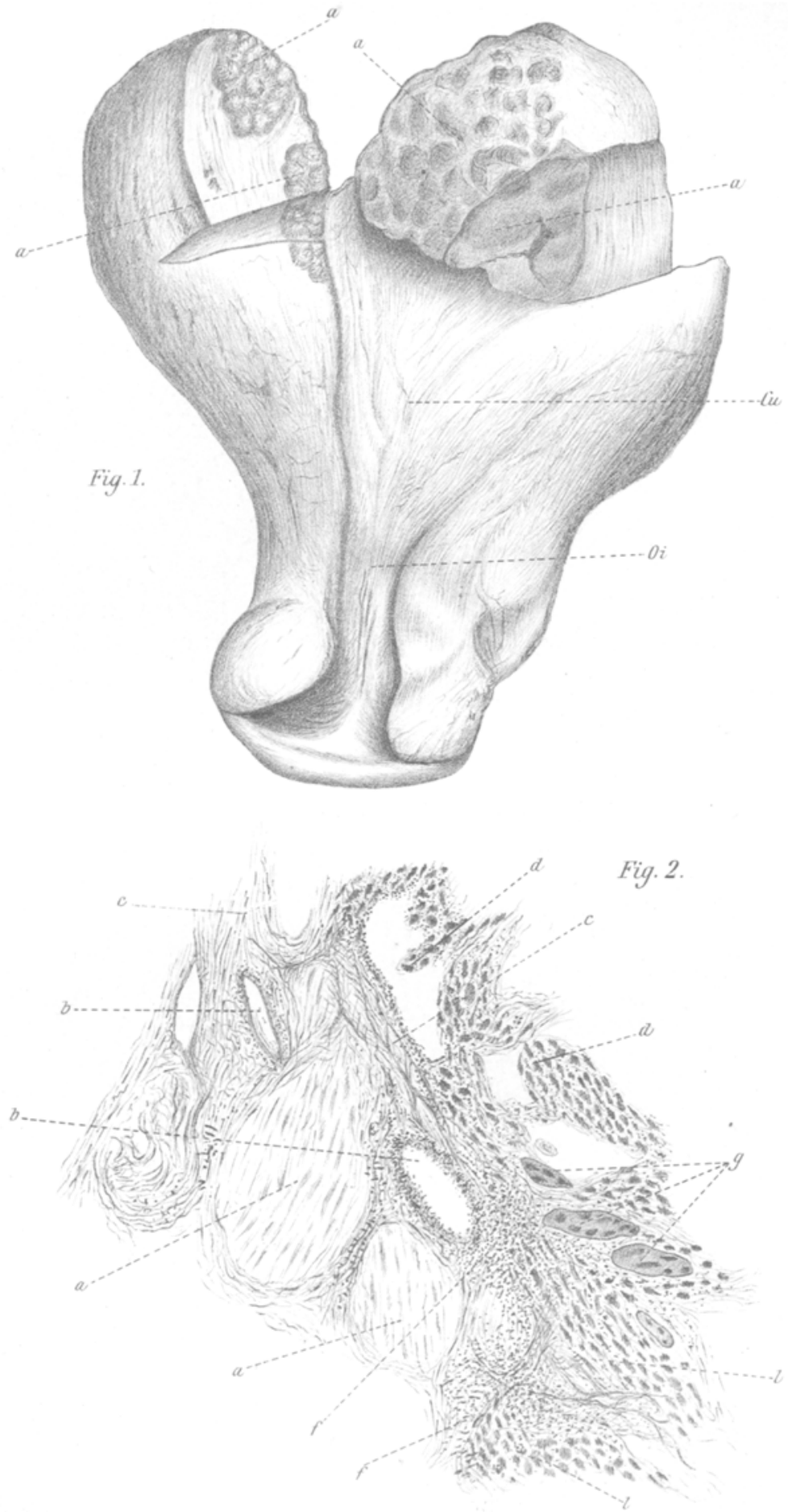